\title{
Does FeNO Predict Clinical Characteristics in Chronic Cough?
}

\author{
Mahboobeh Haji Sadeghi ${ }^{1}$ (1) . Caroline E. Wright ${ }^{1} \cdot$ Simon Hart $^{1} \cdot$ Michael Crooks $^{1} \cdot$ Alyn H. Morice $^{1,2}$
}

Received: 21 September 2017 / Accepted: 17 November 2017 / Published online: 25 November 2017

(c) The Author(s) 2017. This article is an open access publication

\begin{abstract}
Purpose To evaluate whether exhaled nitric oxide measurement can facilitate in the assessment of chronic cough patients based on their airway inflammatory phenotype.

Methods We have studied consecutive patients attending a specialist cough clinic. 30 patients with high FeNO ( $>30 \mathrm{ppb})$ and 20 patients with low FeNO $(<20 \mathrm{ppb})$ were recruited.

Results There was a significant correlation between FeNO, B-Eos and sputum eosinophil count $(p<0.001)$. The number of recorded coughs in $24 \mathrm{~h}$ and HARQ scores were significantly $(p<0.05)$ higher in patients with a low FeNO. In contrast to the high FeNO group (48\%), the greater proportion of these patients were women (90\%). LCQ scores were worse in the low FeNO group but it was not significant.

Conclusion A strong relationship between FeNO, blood eosinophils and sputum eosinophils confirming phenotypic identity was observed. Whether the observed gender disparity accounts for the different cough frequency characteristics is unknown.
\end{abstract}

Keywords Chronic cough $\cdot$ FeNO $\cdot$ Airway inflammation

\section{Introduction}

The diagnosis of chronic cough is controversial with different terms being used to describe similar clinical presentations. Recently, a unifying diagnosis of cough hypersensitivity has been proposed with treatment dependent on the type of airway inflammation present. How best to evaluate the inflammatory phenotype in a patient with chronic cough has been studied using fractional exhaled nitric oxide (FeNO) measurement [1-5]. However, the different clinical phenotype of patients with chronic cough based on their inflammatory profiles has not been studied in depth. We therefore divided sequential patients attending a specialist cough clinic into two groups of low FeNO (FeNO $\leq 20 \mathrm{ppb})$ and high $\mathrm{FeNO}$ (FeNO $\geq 30 \mathrm{ppb}$ ) to evaluate the profile of other eosinophilic biomarkers, cough frequency and demographics to determine if they exhibited phenotypic variability.

Alyn H. Morice

A.H.Morice@ hull.ac.uk

1 Respiratory Medicine, Castle Hill Hospital, Institute for Clinical and Applied Health Research (ICAHR), Hull York Medical School, Cottingham, UK

2 Castle Hill Hospital, Hull York Medical School, Cottingham HU16 5JQ, UK

\section{Methods}

\section{Study Design}

In this study, we aimed to explore the efficacy of FeNO measurement in determining airway inflammatory phenotype in chronic cough patients. Correlation between FeNO, blood and sputum eosinophil cell count was assessed. We then determined the objective and subjective measurements of cough in patients with high FeNO and low FeNO. 24-h cough counts were measured using the Hull Automated Cough Counter (HACC). Hull Airways Reflux Questionnaire (HARQ) and Leicester Cough Questionnaire (LCQ) were applied to measure cough subjectively.

Patients with a history of chronic cough for more than an 8 -week duration were recruited sequentially from the Hull Cough Clinic. Subjects excluded from the study were those with a current diagnosis of classic asthma, patients who were suffering from any significant concomitant disease, a lower respiratory tract infection in the last 4 weeks, subjects who were taking Angiotensin-Converting Enzyme (ACE) inhibitors and current smokers. The concomitant use of inhaled corticosteroids or bronchodilators was allowed provided that dosing was stable for at least 4 weeks prior to enrolment 
(none of the patients in the low FeNO group were using inhaled corticosteroids).

After informed consent was obtained, FeNO, spirometry, sputum collection, full blood count, 24-h cough count, HARQ and LCQ were performed.

The study was approved by a local ethical review committee (EudraCT No: 2015-001736-38) and registered with Clinicaltrials.gov (No: NCT02479074).

\section{Methodology}

FeNO was measured with a NIOX VERO machine supplied by Aerocrine Ltd. at an expiratory flow rate of $50 \mathrm{~mL} / \mathrm{s}$, according to the ATS and ERS recommendations [6]. A calibrated electrochemical sensor analyses the last $3 \mathrm{~s}$ of the 10-s exhalation to indicate results in parts per billion (ppb) with a measurement range of 5-300 ppb. FENO less than $25 \mathrm{ppb}$ in adults is less likely to indicate eosinophilic inflammation and response to corticosteroids [7].

A pneumotach within KoKo Spirometer were used to measure lung function according to the specifications and performance criteria recommended by the American Thoracic Society (ATS)/European Respiratory Society (ERS) Standardization of Spirometry [8].

The Hull Automated Cough Counter (HACC) and Leicester Cough Monitor (LCM) software were used to measure the cough frequency over a $24-\mathrm{h}$ period. The automated assessment of cough is valid, reliable and highly reproducible $[9,10]$ and significantly correlated with subjective assessment of cough and cough reflex sensitivity [11].

Sputum samples were collected by applying different techniques such as spontaneous expectoration or sputum induction. DeVilbiss UltraNeb Ultrasonic Nebuliser with an average output of $1 \mathrm{ml} / \mathrm{min}$ was used to generate aerosols with a dose of about 5-7 mL per inhalation to collect induced sputum [12]. The device was set according to the Standard Operating Procedure of the Clinical Trial Unit No: CTU101099. The Standard Operating Procedure of the Clinical Trial Unit SOPCTU100210 has been used to process the sputum samples, while some minor alterations have been applied.

HARQ and LCQ were used as subjective measures of cough. HARQ is a 14-point questionnaire, each question independently testing for the cough hypersensitivity syndrome on a scale of $0-5(0$, no problem; 5 , severe/frequent problems), with the total score varying from 0 to 70 points, and the upper limit of normal is 13 out of 70 . The LCQ contains 19 questions to assess three domains of physical, psychological and social with a seven-point Likert response scale, ranging from $1=$ all of the time to $7=$ none of the time . The score varies from 3 to 21 , a higher score indicated better quality of life and a change of 2.56 in total LCQ score is more likely to be significant [13].

\section{Statistical Analysis}

Subjects' ages and gender, FeNO, 24-h cough count, LCQ and HARQ questionnaires, spirometry measurement, sputum eosinophilic count and blood eosinophil count (B-Eos) data were expressed as a mean $\pm(\mathrm{SD})$ using SPSS Descriptive statistic test.

ANOVA test was used to compare changes in the mean FeNO value, number of coughs in $24 \mathrm{~h}$, sputum eosinophil cell count, spirometry measurements, B-Eos, HARQ and LCQ scores between the low FeNO group and the high FeNO group. $\mathrm{P}$ value $<0.05$ was considered significant.

Pearson's correlation coefficient $(r)$ test was used to evaluate the correlation between FeNO, B-Eos and sputum eosinophil cell count.

\section{Results}

\section{Demographics}

It was planned to recruit 60 chronic cough patients, 40 with a FeNO $\geq 30 \mathrm{ppb}$ and 20 with a FeNO $\leq 20 \mathrm{ppb}$. However, patients with high FeNO represented only $10 \%$ of the clinical population at the time of the study, and because of slow recruitment we enrolled only 30 patients in this group. In total, 50 patients were recruited into the study, 30 patients in the high FeNO group and 20 patients in the low FeNO group. One patient was withdrawn from the study due to an error in the randomisation. In total, 49 patients were enrolled to the study, 29 in the high FeNO and 20 in the low FeNO group. Mean ( \pm SD) age was $62 \pm 9.5$ (range $45-82$ years). $32(65 \%)$ of the subjects were female. There was a marked gender difference between the two groups. The low FeNO group comprised $90 \%$ women (18 women and 2 men), whereas the sexes were almost equally represented in the high FeNO group (15 men and 14 women). There was no evidence of airflow obstruction with FEV1 being 96\% predicted in the high FeNO and $113 \%$ in the low FeNO value (NS).

\section{Airway Inflammatory Biomarkers (FeNO Value, Blood and Sputum Eosinophil Count)}

Unsurprisingly, there was a significant difference in mean FeNO value between the high FeNO $(65 \pm 39 \mathrm{ppb})$ and low FeNO $(13 \pm 5 \mathrm{ppb})$ groups $(p<0.005)$. Mean B-Eos in the high FeNO group was $0.34 \pm 0.2 \times 10^{9} / \mathrm{L}$, whereas in the low FeNO group it was $0.16 \pm 0.1 \times 10^{9} / \mathrm{L}(p<0.005)$. In the high FeNO group, half of the patients (14) had a B-Eos above $0.3 \times 10^{9} / \mathrm{L}$, whereas the rest had a B-Eos between 0.2 
and $0.1 \times 10^{9} / \mathrm{L}$. In the low FeNO group, all the patients had a B-Eos under $0.3 \times 10^{9} / \mathrm{L}$, and only a single patient had a high B-Eos of $0.56 \times 10^{9} / \mathrm{L}$ (Fig. 1).

In 30 patients (15 in the high and 15 in the low FeNO group) who had a previous blood test (median $=4$ months, range $=1$ month to 26 months) in their clinical record B-Eos results were compared. The mean current B-Eos were highly correlated $(r=0.64, p<0.001)$ with the previous B-Eos. Thus, the majority of the patients in the high FeNO group had a previous history of high blood eosinophilic inflammation. Bland-Altman analysis revealed that this correlation declined at higher blood eosinophil counts (Fig. 2).

Thirty sputum samples were successfully processed and counted. The mean eosinophil cell counted in sputum samples in the high FeNO group was $15 \pm 26 \%$, while in the low FeNO group it was $0.4 \pm 0.6 \%(p<0.05$ equal variances not assumed). Patients with low FeNO all had eosinophil cell count under $0.5 \%$, except one whose eosinophil cell count was $2 \%$ which is within the laboratory normal range $(<3 \%)$. Half of the patients in the high FeNO group had an eosinophil cell count under 3\%. However, almost all of them had eosinophil cell count above $0.5 \%$ except two with $0 \%$
(Fig. 3). Percentage of macrophages in low FeNO patients $(65 \%)$ was significantly higher $(p<0.05)$ compared with the patients with high FeNO (36\%). Other inflammatory cell counts in sputum samples such as neutrophils, epithelial and lymphocytes were similar in both cohorts.

In the 30 patients who had FeNO, B-Eos and sputum eosinophil count, a strong correlation was observed with FeNO $r=0.79$ and $r=0.65, p<0.001$, respectively. The correlation between B-Eos and sputum eosinophil count was more modest $r=0.59, p<0.001$.

\section{Objective and Subjective Measurements of Cough (24-h Cough Count, LCQ and HARQ)}

Forty-eight patients, 20 in the low FeNO group and 28 in the high FeNO group, completed 24-h cough count measurement (device failure led to loss of data on two occasions). There was a highly significant difference $(p<0.005)$ between the high and low FeNO groups in the number of recorded coughs in $24 \mathrm{~h}$. The mean number of coughs in $24 \mathrm{~h}$ in the low FeNO group was $540 \pm 376$, whereas this was $270 \pm 220$ in the other group. A similar significant difference

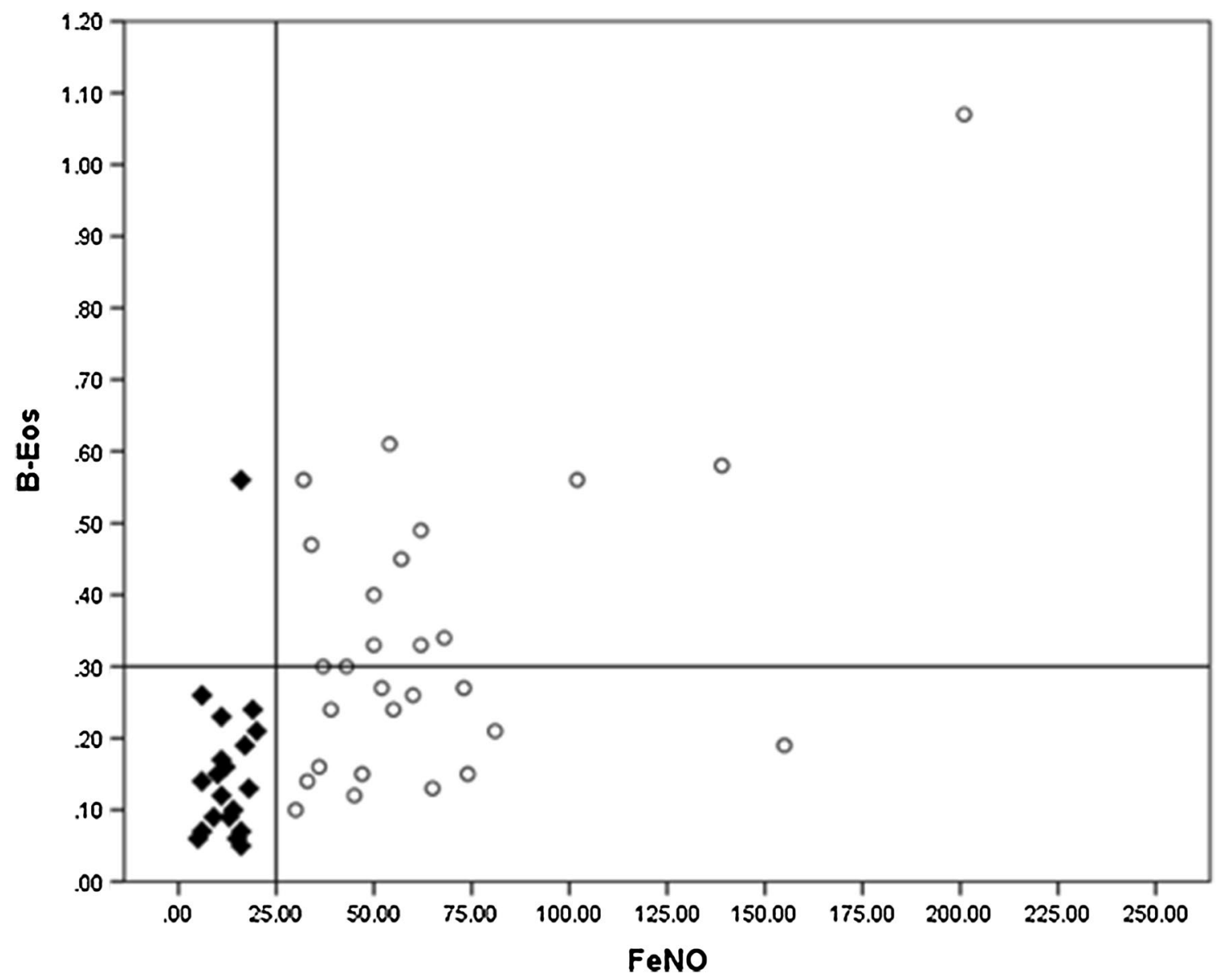

Fig. 1 Scatter plot of FeNO ppb and B-Eos $\times 10^{9} /$ L. Filled triangle: Low FeNO group. Circle: High FeNO group 


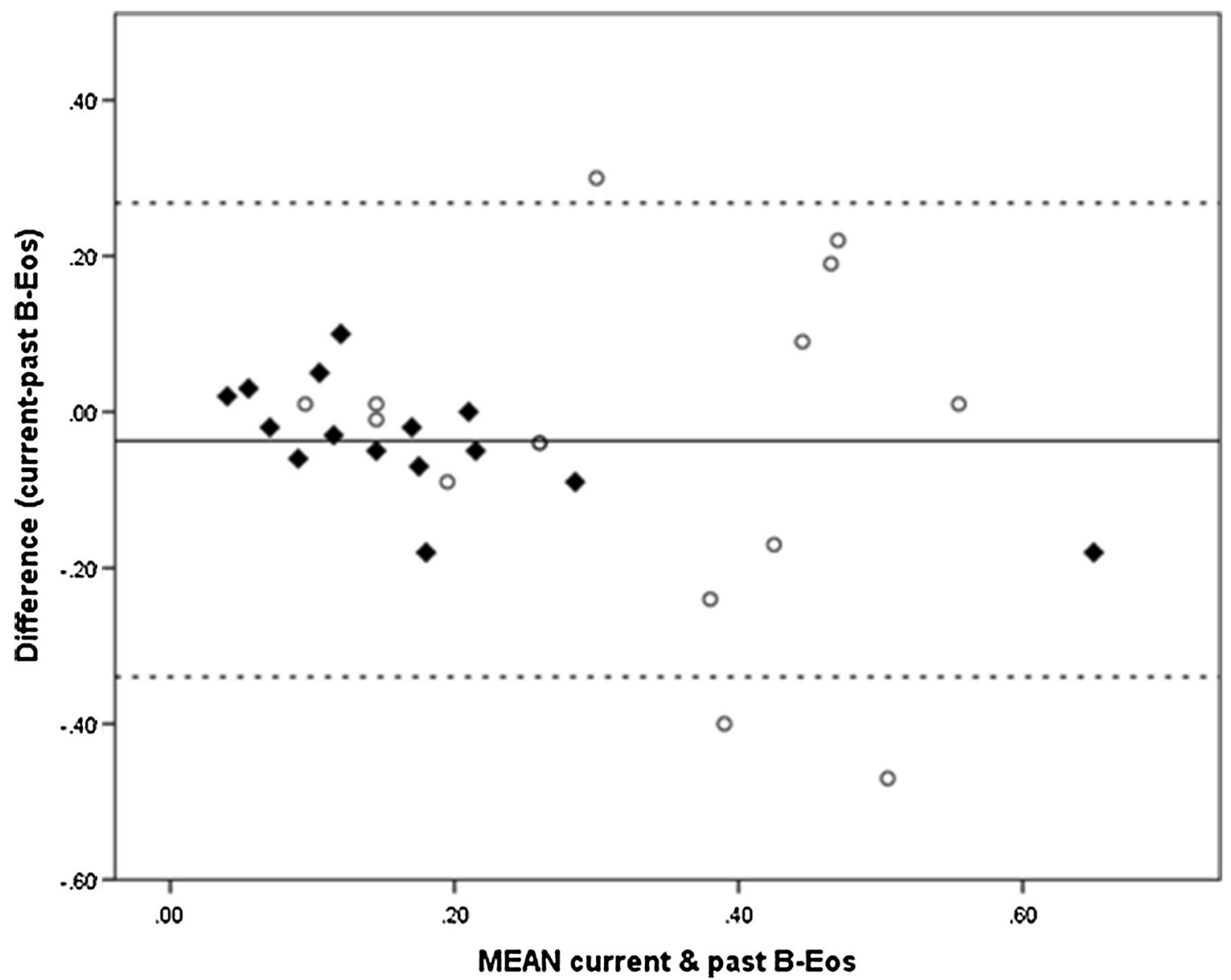

Fig. 2 Bland-Altman plot of current B-Eos and previous B-Eos. Filled triangle: Low FeNO group. Circle: High FeNO group

$(p<0.05)$ in the HARQ score between the two cohorts was observed. The mean HARQ score was $39 \pm 12$ in the low FeNO group, whereas it was $32 \pm 11$ in the high FeNO group. The LCQ scores in the low and high FeNO groups on average were $12 \pm 4$ and $14 \pm 3$, respectively; however, this did not achieve statistical significance. Overall, patients with low FeNO suffered greater morbidity in comparison with patients with high FeNO as assessed by 24-h cough count, HARQ and LCQ.

\section{Discussion}

We have evaluated the demographic data, 24-h cough count, HARQ and LCQ in sequentially recruited patients attending a specialist cough clinic. Patients were stratified into the high FeNO and low FeNO groups and the different characteristics of these two cohorts were observed.

In contrast to our investigation of unselected patients attending a cough clinic, others have studied the inflammatory profile of patients with a variety of diagnoses such as cough variant asthma and forms of eosinophilic bronchitis.
Whether such conditions are separate disease entities or part of the inflammatory continuum of cough hypersensitivity syndrome is controversial [14]. In none of these studies was cough objectively assessed.

Chatkin et al. [1] determined FeNO values in patients with cough of more than 3 weeks and found that those with bronchial hyperresponsiveness and FeNO $>30$ ppb were more likely to be diagnosed as asthmatic on review. In another study, patients with cough of more than 3 weeks were classified into three groups of asthmatic cough, nonasthmatic eosinophilic bronchitis (NAEB) and "others" based on spirometric reversibility, methacholine responsiveness and sputum eosinophilia [3]. They found FeNO values lower than $31 \mathrm{ppb}$ indicating that asthma and NAEB were unlikely. Maniscalco and colleagues [4] assessed patients with cough of more than 8 weeks and classified them into four categories of cough variant asthma (CVA), NAEB, gastroesophageal reflux disease (GERD) and upper airway cough syndrome (UACS) according to the ACCP guidelines [15]. They reported that the mean FeNO values in CVA and NAEB were more than double those in UACS and GERD. Thus, in various groups of cough patients low 


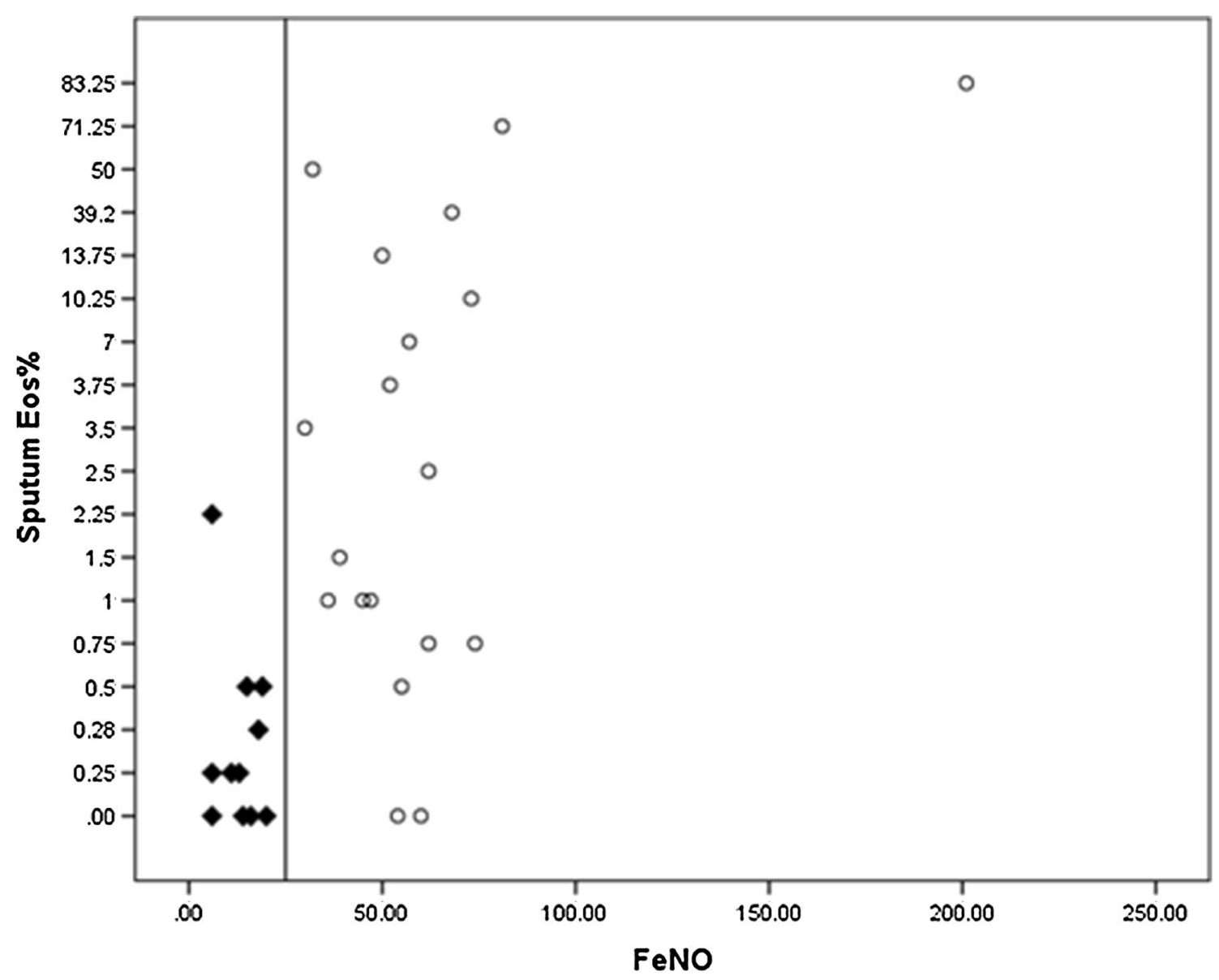

Fig. 3 Scatter plot of FeNO ppb and sputum Eos\%. Filled triangle: Low FeNO group. Circle: High FeNO group

and high FeNO values have been associated with a different airway inflammatory profile; however, the effect on cough frequency has not been examined.

In our study, cough frequency in the low FeNO group was double that seen in the high FeNO group and this was associated with a greater impact on quality of life as assessed by the LCQ and HARQ. When the pattern of coughing was examined, there was no discernible difference between the groups, both exhibiting the well-described abatement of cough during sleep. While the airway inflammatory profiles and cough frequency differences between the two groups are important, there was a mismatch between the sexes. Patients in the low FeNO group were predominantly women, whereas the high FeNO group had a similar sex distribution. Interestingly, a similar disparity was seen in a previous study [3]. Experience of cough clinics around the globe suggests that there is a 2-to-1 preponderance of women attending cough clinics, possibly reflecting a greater cough reflex sensitivity $[16,17]$, but the possible relationship between gender and different inflammatory profiles has not previously been described. A recent large database study by Price and colleges [18] has shown a similar female gender bias of 1.39 in pauci-eosinophilic asthma. Further investigation in a larger number of cough hypersensitive patients will be required to confirm our findings.

Women patients have been shown to have a greater 24-h cough count than men [19], and since in our low FeNO cohort women predominated this may explain the almost doubling of mean recorded cough seen in the low FeNO group. To demonstrate that this difference resides in the low FeNO inflammatory profile rather than gender require further study with sexual stratification. However, the observed differences in the low FeNO group appear to be genuine as both the scores of HARQ and LCQ were worse in this cohort. If this is confirmed, FeNO may be clinically useful in predicting inflammatory phenotypes cough hypersensitivity.

In this study, we found a high degree of correlation between the different measures of airway inflammatory biomarkers. Average FeNO value, blood and sputum eosinophil count were markedly different in the low and high FeNO groups, indicating the lack of eosinophil inflammation in the low FeNO group. To our knowledge, this the first study in chronic cough patients which assesses the correlation between FeNO and B-Eos, and it contrasts with studies in 
asthmatic patients where only a modest $(\mathrm{r}=0.51, p<0.001)$ or weak $(\mathrm{r}=0.22, p<0.001)$ correlation between FeNO value and B-Eos was reported [20, 21]. Thus, these biomarkers may have a different profile in chronic cough patients. Our study is consistent with previous observations in cough [5] and asthmatic $[4,22]$ patients, which have shown that FeNO has a strong correlation with sputum eosinophil count. The correlation between B-Eos and sputum eosinophil count was modest in our study and similar observations were reported in an asthma study [22].

In conclusion, we showed a meaningful relationship between FeNO, blood eosinophils and sputum eosinophils in chronic cough. Our data indicate that we may use FeNO to phenotype these patients and this may be of therapeutic relevance.

Acknowledgements This work was funded by the Aerocrine Limited Company. The investigators were independently responsible for the study protocol, study design, methods, data collection, data analysis and interpretation, including statistical reports and figures.

\section{Compliance with Ethical Standards}

Conflict of interest The authors declare that they have no competing interests.

Open Access This article is distributed under the terms of the Creative Commons Attribution 4.0 International License (http://creativecommons.org/licenses/by/4.0/), which permits unrestricted use, distribution, and reproduction in any medium, provided you give appropriate credit to the original author(s) and the source, provide a link to the Creative Commons license, and indicate if changes were made.

\section{References}

1. Chatkin JM et al (1999) Exhaled nitric oxide as a noninvasive assessment of chronic cough 159(6):1810-1813

2. Fujimura $\mathrm{M}$ et al (2008) Exhaled nitric oxide levels in patients with atopic cough and cough variant asthma. Respirology 13(3):359-364

3. Oh M-J et al (2008) Exhaled nitric oxide measurement is useful for the exclusion of nonasthmatic eosinophilic bronchitis in patients with chronic cough. Chest 134(5):990-995

4. Maniscalco $M$ et al (2015) Extended analysis of exhaled and nasal nitric oxide for the evaluation of chronic cough. Respir Med 109(8):970-974
5. Yi F et al (2016) Validity of fractional exhaled nitric oxide in diagnosis of corticosteroid-responsive cough. Chest 149(4):1042-1051

6. ATS/ERS Recommendations for standardized procedures for the online and offline measurement of exhaled lower respiratory nitric oxide and nasal nitric oxide, 2005. Am J Respir Crit Care Med 2005 171(8): 912-30

7. Dweik RA et al (2011) An official ATS clinical practice guideline: interpretation of exhaled nitric oxide levels (FENO) for clinical applications. Am J Respir Crit Care Med 184(5):602-615

8. Miller MR et al (2005) Standardisation of spirometry. Eur Respir J 26(2):319-338

9. Barry SJ et al (2006) The automatic recognition and counting of cough. Cough 2:8

10. Birring SS et al (2008) The Leicester Cough Monitor: preliminary validation of an automated cough detection system in chronic cough. Eur Respir J 31(5):1013-1018

11. Faruqi $S$ et al (2011) Quantifying chronic cough: objective versus subjective measurements. Respirology 16(2):314-320

12. Boot JD, Panzner P, Diamant Z (2007) A critical appraisal of methods used in early clinical development of novel drugs for the treatment of asthma. Pulm Pharmacol Ther 20(3):201-219

13. Birring $\mathrm{S}$ et al (2003) Development of a symptom specific health status measure for patients with chronic cough: Leicester Cough Questionnaire (LCQ). Thorax 58(4):339-343

14. McGarvey L, Morice AH (2003) Atopic cough: little evidence to support a new clinical entity. Thorax 58(8):736-737 author reply 737-8.

15. Irwin $\mathrm{R}$ et al., Diagnosis and management of cough executive summary: ACCP evidence-based clinical practice guidelines. Chest 2006 129:1S-23S

16. Morice AH et al (2014) A worldwide survey of chronic cough: a manifestation of enhanced somatosensory response. Eur Respir J 44(5):1149-1155

17. Kastelik JA et al (2002) Sex-related differences in cough reflex sensitivity in patients with chronic cough. Am J Respir Crit Care Med 166(7):961-964

18. Price DB et al (2015) Blood eosinophil count and prospective annual asthma disease burden: a UK cohort study. Lancet Respir Med 3(11):849-858

19. Kelsall A et al (2009) Sex differences and predictors of objective cough frequency in chronic cough. Thorax 64(5):393-398

20. Strunk RC et al (2003) Relationship of exhaled nitric oxide to clinical and inflammatory markers of persistent asthma in children. J Allergy Clin Immunol 112(5):883-892

21. Malinovschi A et al (2013) Exhaled nitric oxide levels and blood eosinophil counts independently associate with wheeze and asthma events in National Health and Nutrition Examination Survey subjects. J Allergy Clin Immunol 132(4):821-827.e1-5

22. Wagener AH et al (2015) External validation of blood eosinophils, $\mathrm{FE}(\mathrm{NO})$ and serum periostin as surrogates for sputum eosinophils in asthma. Thorax 70(2):115-120 